\title{
The role of zinc in the treatment of palm kernel cake toxicity in sheep
}

\author{
M Hair-Bejo, AR Alimon, J Maria, MY Hass, M Moonatizad \\ Faculty of Veterinary Medicine and Animal Science, Universiti Pertanian Malaysia, 43400 UPM \\ Serdang, Selangor, Malaysia
}

Palm kernel cake (PKC), a product of the oil palm industry, has a greater potential as a major feed ingredients in ruminants. However, the high content of copper in PKC can induce chronic copper toxicity in sheep. Death is mainly due to hepatic necrosis resulting from excessive hepatic copper accumulation, but it can be prevented by dietary zinc supplementation (Hair-Bejo and Alimon, 1992, Proc 15th MSAP Ann Conf, 93-95). However, the role of zinc in the treatment of PKC toxicity is little understood. Thus, it is the aim of the study to determine the role of zinc in the removal of copper in PKC fed sheep.

Twenty five, 4-month-old male Malin cross sheep were allocated into 5 groups of 5 animals each. Three groups of animals were fed with PKC $(90 \%)$ and grass $(10 \%)$ for 16 weeks before they were slaughtered (PKC-I) or further fed with the diet either with $(P K C+Z n)$ or without (PKC-1I) zinc supplementation (500 $\mu \mathrm{g} / \mathrm{g} \mathrm{Zn}$ as zinc sulfate) for another 16 weeks. The control groups were fed with corn and fish meals $(40 \%)$ and grass $(60 \%)$, and were slaughtered at weeks 16 and 32 of the trial. The blood, right liver, renal cortex, bile, urine and pancreas were analysed for copper and zinc contents using an Atomic Absorption Spectrophotometry. The metal contents were expressed as mean \pm SEM in $\mu \mathrm{g} / \mathrm{g}$ dry weight or $\mu \mathrm{g} / \mathrm{ml}$, whilst statistical analysis was performed using Student's t-test.

The study showed that neither clinical signs nor gross lesions of chronic copper or zinc toxicity were observed throughout the trial. However, the copper content in the right liver of sheep fed PKC at week $16(1716.9 \pm 677.9)$ rose to about 3 times $(P<0.05)$ then the control group (487.5 \pm 73.5$)$ and remained unchanged $(P>0.05)$ thereafter in both the PKC-II (1813.2 $\pm 606.7)$ and $P K C+Z n$ groups (1886.6 \pm 1255.8). A similar pattern of copper content was observed in the blood. The zinc content in the liver of $P K C+Z n$ group $(205.1 \pm 44.6)$ increased $(P<0.05)$ when compared to those of the control (166.6 \pm 39.5$)$ and PKC-ll groups (158.9 \pm 47.7$)$. However, the copper and zinc contents in renal cortex, bile, urine, and pancreas remained unchanged $(P>0.05)$ in all groups throughout the trial.

The zinc treatment failed to remove copper which had been accumulated in the liver. In addition, the absent of any increase in the bile, renal cortex and urine copper contents suggest that zinc does not facilitate copper removal through this routes. This further support the previous finding which showed that the antagonistic interaction between copper and zinc occurs at the luminal level of the intestinal tract (Hair-Bejo et al, 1991, Proc 3rd VAM Con, 59-61). The ability of sheep in the present trial to tolerate to potentially toxic effect of copper in PKC (23.4 \pm 0.8$)$ is an interesting phenomenon, although it has been observed previously in goats (Hair-Bejo et a/, 1993, Proc 5th VAM Con, 63-64) and cattle (Hair-Bejo ef al, 1994, Int Con QVS 21th Century, 262).

It was concluded that the usage of dietary zinc supplementation in the treatment of PKC toxicity in sheep is unsatisfactory, whilst the ability of sheep to tolerate high copper content in PKC needs further clarification. 